\title{
CHEMICAL AND ELECTRON BEAM REDUCTION OF VANADIUM OXIDES MONITORED BY EELS AND NEXAFS
}

\author{
D.S. Su, M. Hävecker, M. Willinger, R. Schlögl \\ Department of Inorganic Chemistry, Fritz-Haber-Institute, Max-Planck Society, \\ Faradayweg 4-6, D-14195 Berlin, Germany
}

The application of modern transmission electron microscopes (TEM) in catalytic research has accelerated the progress in understanding the mechanism of catalytic reactions. For instance, using environmental TEM, the novel glide shear defect process was revealed as an efficient mechanism for the release of the structural oxygen of vanadyl pyrophosphate in the $n$-butane oxidation [1]. On the other hand, electron energy-loss spectroscopy (EELS), which is nowadays commercially available on modern TEM, appears unknown in the catalytic community. In fact, core-loss EELS is a powerful tool to identify the chemical state of elements and to study the modification of this sate in various compounds.

Fig.1 shows EELS-spectra of various vanadium oxides in which the oxidation state of vanadium varies from 2+ (VO) to 5+ $\left(\mathrm{V}_{2} \mathrm{O}_{5}\right)$. The first two features, marked as $\mathrm{V} \mathrm{L}_{3}$ and $\mathrm{V} \mathrm{L}_{2}$ edges, are attributed to the excitations from $\mathrm{V} 2 p_{3 / 2}$ and $\mathrm{V} 2 p_{1 / 2}$ core levels to the unoccupied $\mathrm{V} 3 d$ states, respectively. The third peak, marked by $\mathrm{O} K$, is due to the excitation of $\mathrm{O} 1 s$ electrons. Since the local surrounding of a vanadium atom in the oxides determines the charge distribution of valence band, the effective potential on that atom causes the change of all the electronic levels. Therefore the difference in the oxidation state of vanadium results in chemical shifts of $\mathrm{V} \mathrm{L}_{3,2}$ edges in EELS spectra, as it is shown in Fig.1. The fine structure of oxygen $\mathrm{K}$-edge gives the fingerprint of the bonding properties of vanadium such as the degree of the covalence and the hybridisation $\mathrm{V}$ $3 d-\mathrm{O} 2 p$ orbitals for high valence vanadium.

The reductive behaviour of $\mathrm{V}_{2} \mathrm{O}_{5}$ in an inert, simple ambient high-vacuum, induced by electron beam in a Philip CM 20 electron microscope, is studied by means of EELS. Spectra recorded after various irradiation times are shown in Fig. 2. The similarity of the spectra in Fig. 1 and 2 becomes obvious. The chemical shift of $\mathrm{V} \mathrm{L}_{3,2}$ edges reveals the reduction of $\mathrm{V}_{2} \mathrm{O}_{5}$ to $\mathrm{VO}$.

The obtained results can be used for the comparison with NEXAFS. An apparatus which allows to take in situ X-ray absorption spectra in the soft energy range $(150 \mathrm{eV}$ hí $1000 \mathrm{eV})$ under pressures in the mbar range was developed [2]. Fig. 3 shows the NEXAFS of the V L-edges and the $\mathrm{O}$ K-edge of a $\mathrm{V}_{2} \mathrm{O}_{5}$ pellet (pressed powder) before (A) and during the reduction in $0.30 \mathrm{mbar}$ methanol at $623 \mathrm{~K}(\mathrm{~B})$, together with the spectra taken in vacuum after this treatment $(\mathrm{C})$ and a spectrum of $\mathrm{V}_{2} \mathrm{O}_{5}$. Comparison of NEXAFS with EELS-spectra in Fig.1 and 2 indicates a partial reduction of vanadium already in the $\mathrm{V}_{2} \mathrm{O}_{5}$ pellet. The final state of vanadium after chemical treatment in methanol was identical to that of $\mathrm{V}_{2} \mathrm{O}_{5}$ after reduction under high vacuum induced by the electron beam. This finding will be discussed in the context of "lattice oxygen" for catalysis.

\section{References}

[1] P.L. Gai, Topics in Catalysis 8 (1999) 97

[2] A. Knop-Gericke, M. Hävecker, T. Neisius, T. Schedel-Niedrig, Nucl. Instr. Meth. A 406

(1998) 311 


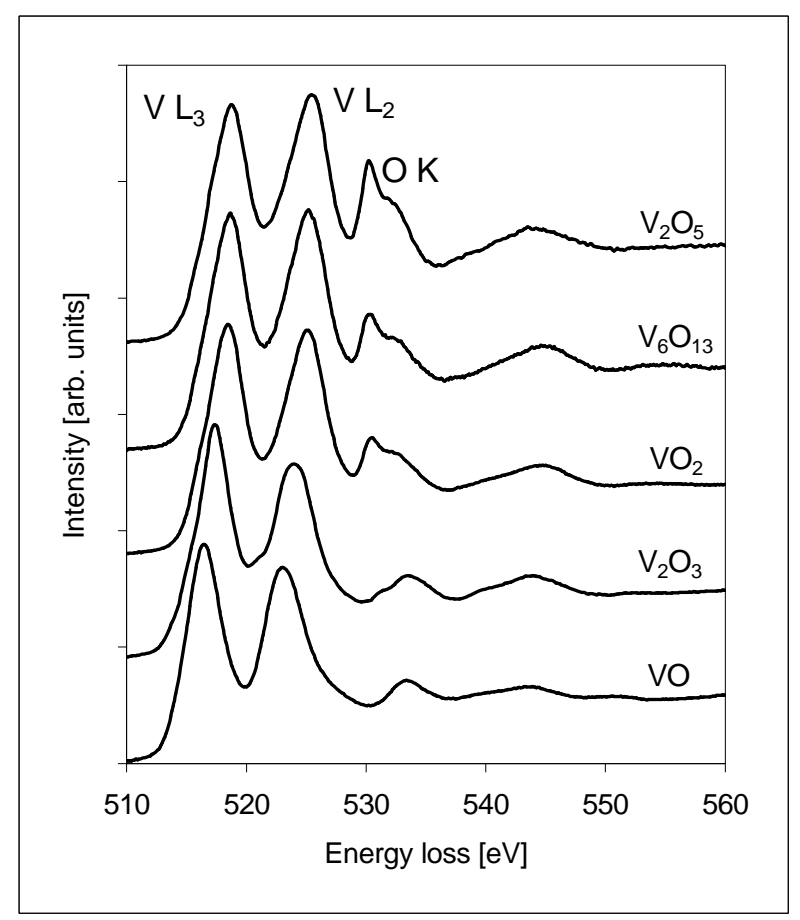

Fig. 1 EELS-spectra of various vanadium oxides recorded on a Philips CM 200 Electron microscope with a GATAN filter GIF 100.

Fig. 2 EELS-spectra of $\mathrm{V}_{2} \mathrm{O}_{5}$ recorded after various periods of irradiation. Current density: 3 $\mathrm{A} / \mathrm{cm}^{2}$.

Fig. 3 NEXAFS of the V L-edges and the O Kedge of a $\mathrm{V}_{2} \mathrm{O}_{5}$ pellet (pressed powder) before (A) and during the reduction in 0.30 mbar methanol at $623 \mathrm{~K}(\mathrm{~B})$. The spectra taken in vacuum after this treatment $(\mathrm{C})$ and the absorption structure of $\mathrm{a}_{2} \mathrm{O}_{5}$ crystal are displayed too.
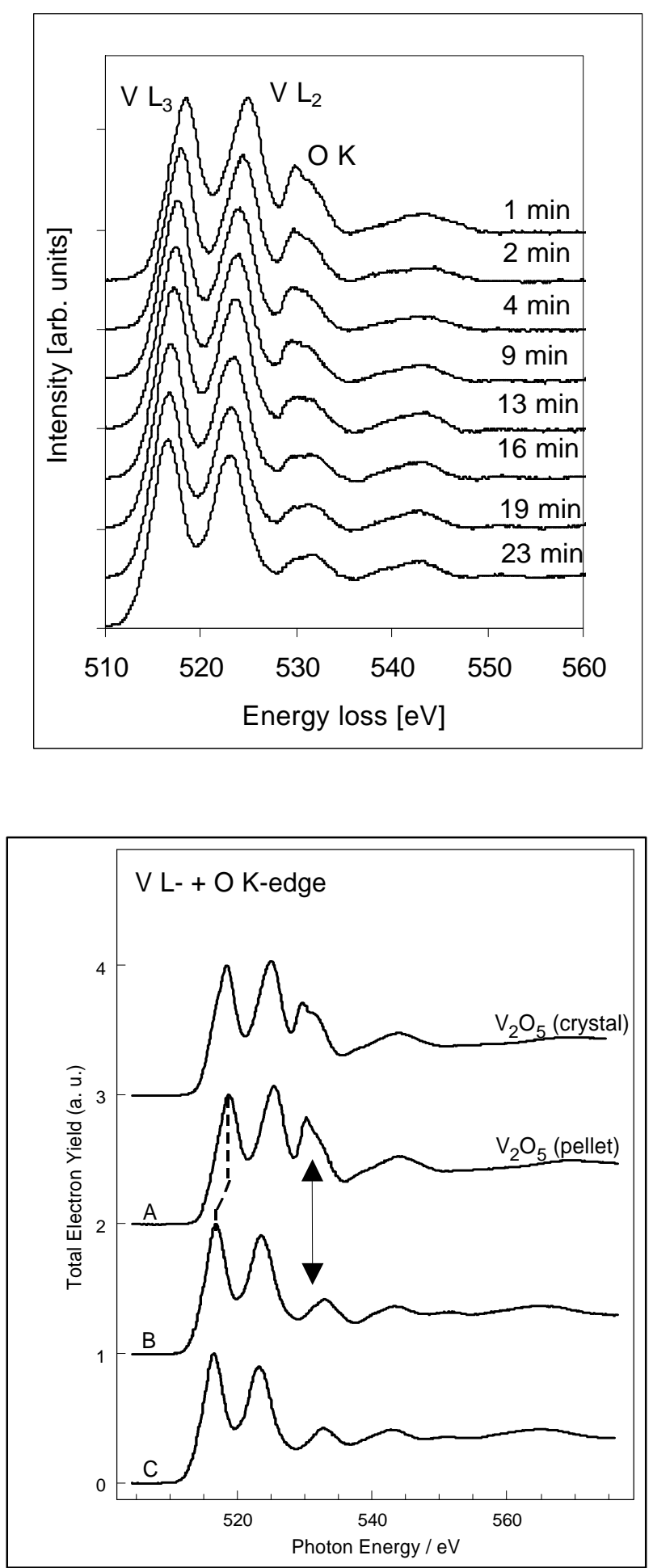

Acknowledgement

The work is supported by SFB 546 of the Deutsche Forschungsgemeinschaft (DFG). 